\title{
Identifying student and teacher difficulties in interpreting atomic spectra using a quantum model of emission and absorption of radiation
}

\author{
Francisco Savall-Alemany \\ Department of General and Specific Didactics, University of Alicante, \\ Sant Vicent del Raspeig, 03690 Alicante, Spain \\ Josep Lluís Domènech-Blanco \\ Department of General and Specific Didactics, University of Alicante, \\ Sant Vicent del Raspeig, 03690 Alicante, Spain \\ Jenaro Guisasola \\ Department of Applied Physics, Polytechnic University College of Donostia, \\ University of the Basque Country, 20018 San Sebastian, Spain \\ Joaquín Martínez-Torregrosa \\ Department of General and Specific Didactics, University of Alicante, \\ Sant Vicent del Raspeig, 03690 Alicante, Spain \\ and Instituto Universitario de Física Aplicada a las Ciencias y a la Tecnología, \\ Sant Vicent del Raspeig, 03690 Alicante, Spain \\ (Received 11 August 2015; published 25 May 2016)
}

\begin{abstract}
Our study sets out to identify the difficulties that high school students, teachers, and university students encounter when trying to explain atomic spectra. To do so, we identify the key concepts that any quantum model for the emission and absorption of electromagnetic radiation must include to account for the gas spectra and we then design two questionnaires, one for teachers and the other for students. By analyzing the responses, we conclude that (i) teachers lack a quantum model for the emission and absorption of electromagnetic radiation capable of explaining the spectra, (ii) teachers and students share the same difficulties, and (iii) these difficulties concern the model of the atom, the model of radiation, and the model of the interaction between them.
\end{abstract}

DOI: 10.1103/PhysRevPhysEducRes.12.010132

\section{INTRODUCTION}

Quantum absorption and emission of electromagnetic radiation, as well as atomic spectra, are fundamental concepts in science with a wide range of technological applications. The history of science shows that at the end of the nineteenth century there was no explanation for some radiation-related phenomena using the classical models of emission and absorption of electromagnetic waves, the spectra of gases being among these phenomena [1,2]. It is not strange, therefore, that the importance of the study of spectra in introductory quantum physics courses has been recognized by school curriculum designers, including spectra phenomena as a fundamental topic, together with other quantum phenomena involving the emission and absorption of electromagnetic radiation, such as the photoelectric effect.

Published by the American Physical Society under the terms of the Creative Commons Attribution 3.0 License. Further distribution of this work must maintain attribution to the author(s) and the published article's title, journal citation, and DOI.
The conventional approach to teaching quantum physics in introductory physics courses at senior high school and university is characterized by a quick and superficial review of the history of physics in the early twentieth century, with special emphasis on certain "quantum topics" such as the photoelectric effect, atomic models, and wave function properties, an emphasis that has not changed since the 1930s [3,4]. Most education research on the teaching of quantum physics has focused on introductory physics courses, addressing topics such as mathematical formalism [5-8], the ontological differences between classical and quantum physics [9-11], and students' interpretations of quantum phenomena [12-15]. However, today there is no consensus on key issues, such as the role that mathematical formalism plays, whether or not to study this formalism in foundation courses, the methodological orientation, or the role of classical physics (or even of the Bohr atomic model) [16-19].

Although physics education research (PER) has recognized the importance of stressing the difficulties that classical physics faced to explain some experimental phenomena, which led to the establishment of quantum 
physics [20,21], there has been little research on how students and teachers interpret atomic spectra. In our opinion, atomic spectra constitute a golden opportunity to study the concepts of quantum mechanics that teachers and students tend to form. The interpretation of atomic spectra requires a correct conceptualization of the quantization of energy both in the atom and in electromagnetic radiation, in addition to a model for explaining the interaction between them.

This article is part of a more ambitious project for the development and testing of guided problem-solving materials [22-24] in introductory physics courses to enable students to understand some basic concepts in quantum mechanics. In this article we focus on the difficulties that students encounter when trying to understand quantum emission and absorption of electromagnetic radiation, and the frequency and the intensity of spectral lines. The question that guides this study is: What concepts do high school students and teachers and university students form when they try to grasp and explain the absorption and emission of electromagnetic radiation by gases?

To answer this question, we reviewed the quantum physics theoretical framework and we identified the key concepts to interpret the emission and absorption spectra in introductory physics courses, including an explanation of spectral lines frequencies and intensities. We then designed two questionnaires, one for teachers and the other for students. The answers were analyzed according to the key concepts identified in the responses. Finally, we draw some conclusions and implications for teaching.

\section{SUBTLETIES OF THE EMISSION AND ABSORPTION QUANTUM MODEL}

At the end of the nineteenth century, some phenomena related to the emission and absorption of electromagnetic radiation could not be explained adequately using classical physics based on electromagnetic waves. The energy distribution of the radiation emitted by a blackbody and the discrete spectra of gases are two examples. It was the attempt to explain how electromagnetic radiation interacts with matter that confronted physics with major contradictions and produced a crisis [25].

Every element in the gaseous state has a characteristic line spectrum. This property of all elements led scientists to hypothesize that the spectrum must be related to the atomic structure of the element in question. In fact, one of the aims of the atomic model proposed by Thomson at the beginning of the twentieth century was to explain these spectra, but it failed [26]. It was Niels Bohr who developed the first satisfactory explanation. Bohr's initial aim was to construct an atomic model consistent with the "nuclear atomic system" proposed by Ernest Rutherford in 1911. Initially, Bohr did not take spectra into account, but he realized that the frequencies of the spectral lines could be a guide for his research. In fact, the main achievement of his model was the explanation of the known spectral frequencies of the hydrogen atom. To achieve this, it was necessary to abandon the classical model whereby a charged oscillator emits electromagnetic waves characterized by a frequency that is identical to the oscillation frequency of the emitter system. Bohr attributed the radiation emission of the hydrogen atom to the transitions between stationary atomic states - in which it could vibrate periodically with a well-defined energy and without emitting radiation-and related the frequency of the emitted or absorbed radiation in a single transition to the difference between the initial and final energy states [27].

In spite of Bohr's success in explaining the hydrogen spectral lines frequencies, his original model had to be extended and modified to account for all the spectra characteristics, especially in three aspects:

(i) Bohr postulated, in 1913, that radiation was formed by electromagnetic waves, in contrast to the photon hypothesis proposed by Albert Einstein in 1905. However, after overcoming strong opposition from the scientific community, the quantum model of radiation based on the photon concept was accepted [28-30] and is used today to explain spectra.

(ii) In processes of radiation absorption, transitions from an excited state to a higher energy state are highly improbable [31]. Experimentally, no lines are detected for transitions between excited states in absorption spectra when atoms in their ground state are used due to the very small probability of such processes occurring. Only if the gas temperature is high enough would there be atoms in the second stationary state and lines could be detected in the absorption spectrum matching the transitions from the second stationary state to the higher states [32]. This limitation was not taken into account in Bohr's initial work. If it happened, the hydrogen atom would ionize easily: after making an initial transition from the ground state to the first excited state it would be possible to absorb a second photon and ionize.

(iii) Bohr's predictions were limited to spectral frequencies. The prediction of their intensity was a weakness in his work [33]. A complete model that attempts to explain the emission and absorption of radiation must take into account not only the frequencies of the spectral lines emitted by the atoms but also their intensity. It was Einstein [34] who linked the spectral lines' intensity with the different probability of transition between the stationary atomic states. This probabilistic nature of atomic transitions is a consequence of the existence of the atomic levels with discrete energy, which make it impossible to make predictions about the atom's future behavior [35,36].

Based on this theoretical background, in order to understand a quantum model in introductory physics courses to 
explain the emission and absorption of electromagnetic radiation, one needs to grasp the following key concepts (KCs) [37-42]:

$\mathrm{KC1}$ (atomic model). Energy is quantized in atoms:

$\mathrm{KC} 1.1$. Atoms can only be found in stationary states, characterized by discrete values of energy, in which they do not emit energy. Any energy change entails the transition of the atom from one stationary state to another.

KC2 (light model). Energy is quantized in radiation:

KC2.1 Radiation is composed of photons, understood as indivisible quanta.

$\mathrm{KC} 2.2$ Each photon's energy is proportional to the radiation frequency.

$\mathrm{KC} 2.3$ The radiation intensity is proportional to the number and energy of photons that form it.

$\mathrm{KC} 3$ (emission and absorption model). Every transition is produced by the interaction between a single photon and a single atomic electron:

KC3.1 The frequency of the radiation emitted or absorbed by an atom is proportional to the energy difference between the states from and to which the transition takes place.

KC3.2 Transitions to lower energy states are of a random nature, both in relation to the final state and in relation to the instant they take place.

KC3.3 The spectral lines' intensity is proportional to the number of atomic transitions per unit of time that originates it.

KC3.4 For a group of atoms at the lower energy level, the absorption of a photon produces transitions from that state to an excited state. Transitions from an excited state to another state of higher energy are possible but highly improbable.

The concepts listed above are key learning objectives of any introductory physics course at university or senior high school. We emphasize that these key concepts, although characteristic of the Bohr atomic model, make no reference to a particular atomic model. A transition between stationary states may be viewed as a transition between orbits in the Bohr atomic model or as changes in the electronic configuration of the atom, as it would be interpreted in a more advanced quantum atomic model. In this study, however, we assume that they suffice to comprehend and master the Bohr atomic model and be able to use it to interpret spectra.

\section{PREVIOUS RESEARCH}

Despite the importance of the study of spectra and the widespread teaching of this topic in introductory physics courses (IPCs), there have been relatively few studies of the problems associated with teaching and learning about atomic spectra in IPCs. Zollman, Rebello, and Hogg [43] presented a small selection of interactive materials to work on spectra formation for gases and other sources of light (LEDs, fluorescent, and phosphorescent objects).
They show that by using their materials, successful results in both students' learning and attitudes are obtained.

Sinarcas and Solbes [44] found that Spanish high school students (16-18 years) have difficulties when explaining discontinuous spectra using Bohr's model. They asked the students the open question: "How do you explain discontinuous spectra using the Bohr atomic model?" to assess whether students understand the role of models and find out whether they relate the electron transition between two levels with the corresponding color band in the spectra. They report that only about $20 \%$ of the students could adequately explain the discontinuous spectra using the corresponding electronic transitions between stationary orbits with well-defined energy. These students explained that the emission or absorption of radiation occurs when the electron moves from one state to another, and they established a relationship of proportionality between the radiation frequency and the energy difference between levels. In addition, the researchers found that about half (54\%) of the students did not answer the question and that almost a quarter $(27 \%)$ gave answers that were logically inconsistent. Their study shows the students' difficulties in responding to qualitative questions related to atomic spectra. However, their research does not explain what models have to be learned by students and does not provide any data about the most common difficulties.

Recently, Ivanjek et al. $[45,46]$ investigated undergraduate physics students' understanding of emission spectra in more detail. In particular, they analyzed whether the students were able to relate the spectral lines' wavelengths with the electronic transitions between atomic energy levels. To do so, the students participating in the study were required (1) to state the maximum number of spectral lines that can be generated from the four lowest atomic energy levels, (2) to identify which line is formed in an emission spectrum when a transition takes place between the two closest energy levels, and (3) to state which is the lowest number of energy levels necessary to form an emission spectrum with 11 lines.

Previous studies on students' understanding of physical optics found that students have a lack of understanding of the dual nature of light as a particle and as a wave [47]. Furthermore, students tend to conceive photons as "classical particles" [48]. With regard to the atomic model, it has also been shown that students mix up classical and quantum concepts $[49,50]$. The difficulties identified may influence students' interpretation of atomic spectra, given that an understanding of the nature of light and atomic structure is a major prerequisite for understanding quantum emission and absorption of radiation.

Our study expands the aims of the aforementioned research. In particular, we take into account the absorption of radiation and the intensity of emitted radiation. In addition, we classify students' conceptions according to three distinct categories: the atomic model, the radiation 
(electromagnetic) model, and the interaction model between radiation and matter. We investigate the degree of understanding of issues such as (a) photon indivisibility, (b) absorbed radiation frequency, (c) spectral line intensity, and (d) transitions between the fundamental and excited states, none of which were discussed in the aforementioned studies.

\section{CONTEXT OF THE STUDY AND METHODOLOGY}

Our main aim is to investigate whether final year students in secondary school (senior high school), university students, and high school teachers understand the key quantum model concepts that explain absorption and emission spectra. These key principles are included in many international curriculum standards for introductory physics courses at both the university level and final year of high school (HS). Our sample encompasses undergraduate physics students, final year high school students (18 years old), and high school teachers. We selected this sample because our goal is to identify the persistent difficulties through the different levels of education in teaching introductory quantum physics courses.

The high school attended by the students in our study is an urban school situated in a city of 100000 people less than $100 \mathrm{~km}$ from the provincial capital of Alicante. This kind of urban high school can be considered typical of Spanish high schools. HS students received $4 \mathrm{~h}$ of physics per week during the course. The course format is $2 \mathrm{~h}$ per week of lecture classes, and $2 \mathrm{~h}$ per week of problem sessions. In the course, students do not normally have the opportunity to participate actively and limit themselves to taking notes from the teacher's explanations, both in lectures and in problem sessions. The students' role was limited to asking questions for clarification after teacher explanations. The sample students belonged to two groups and their teachers were chemistry graduates with more than 10 years experience. As it is compulsory in Spain, they received preservice intensive training in pedagogical content knowledge in order to teach science in secondary education (12-18 years old students). In Spain, graduates in chemistry are allowed to teach physics in high school because there are few graduates in physics in secondary education.

The Spanish high school curriculum [51] specifies that HS students must study "The photoelectric effect and discontinuous spectra: The inadequacy of classical physics to explain them." In addition, they must be able to "explain, using the laws of quantum physics, a series of experiences that classical physics is unable to explain, such as discontinuous spectra [...]." The physics syllabus incorporates topics and exercises included in the course textbook. The Spanish textbooks for the last year of high school are similar to international textbooks for college: see, for example, Ref. [52]. The "Quantum Physics" chapter is taught for about 2 weeks of this course. The lectures and problem solving cover the photoelectric effect, photons, matter waves, energy levels and quantum leaps, Bohr's model of atomic quantization, the Bohr hydrogen atom, and the quantum-mechanical hydrogen atom. The part of the program that deals with "Modern Physics," which includes quantum physics, is an important topic in the final year of high school since in the external assessment for students who intend to take science and engineering courses at the university level, $33 \%$ of the topics covered have to do with quantum physics, subatomic physics, and relativity. Hence, teachers teaching at this level consider it important that their students understand quantum physics.

The fourth-year physics students (P) had completed the credits for the first three years, and were specifically studying quantum mechanics in the last year of their degree course (fourth year). Moreover, the fourth-year physics students had taken an introductory physics course in their first year, which had included the topics described for the HS syllabus.

For the students, we designed a written questionnaire consisting of three questions, which are described in Sec. V. Once the questionnaire had been prepared, we conducted a dry run with high school students, which confirmed that students had no problem understanding how the questions were formulated. The aims of every question presented were validated by four teachers (three from high school and one from university). The respondents of the final version of the questionnaire (see Sec. V) were $37 \mathrm{HS}$ students and 34 undergraduate physics students. The same researcher administered the questionnaire to all the groups in the presence of the teacher of the subject. In high school, the questionnaire was given just after the class had finished topics on quantum physics. In the case of undergraduate physics students, the survey was carried out after completion of the first term of the subject of quantum physics, although the contents required for the questionnaire had already been taught. All students completed the questionnaire under examination conditions (without being able to confer with one another) during class time. The teacher gave an introduction to the questionnaire in which the students were informed that we were working on a study to improve the teaching of physics and they were kindly invited to take part in it. It must be reported that the students approached the task seriously and spent at least 20 min responding. Generally speaking, students experienced no comprehension difficulty with regard to the contents of the questionnaire.

Students' responses were analyzed independently by the researchers. The kappa Cohen reliability coefficient averaged 0.83 for the questions, indicating a very significant degree of agreement in the judges' criteria for applying the categories used to interpret the responses. The kappa intrarater reliability coefficient was also calculated for the main researcher 3 weeks later, obtaining a value of 0.87 on average for all the questions, which is satisfactory for a level of confidence of $95 \%$. The responses from the final questionnaire were analyzed by three authors of this 
article. One of the authors performed a preliminary analysis in which the answers were grouped according to the explanations given by students. The groups that emerged were then discussed in a meeting in which each author analyzed a sample representing $10 \%$ of the questionnaires. The original categories were redefined until a consensus was reached. Each researcher then analyzed each questionnaire individually (kappa coefficient 0.82). Finally, a meeting was held in which all the answers were classified and a consensus was reached on the responses where there had been some disagreement ( $7 \%$ of the total).

We designed three questions for interviewing teachers. These were administered to 30 high school teachers with master's degrees in chemistry (19), in physics (5), or in engineering (6). All of the teachers had more than 10 years of teaching experience. All of the teachers received preservice intensive training (approx. 6 months) in pedagogical content knowledge in order to work as teachers of physics and chemistry at the secondary level. The physics teaching-learning course content focuses on topics in classical mechanics and does not usually address issues in the teaching and learning of quantum physics. All of the teachers have also passed the entrance exams. It is compulsory for Spanish teachers to pass an examination about physics and chemistry content knowledge and pedagogical content knowledge (including modern physics) to become inservice teachers. However, the majority of the applicants have a Master's degree in chemistry. Those who have a Master's degree in physics are more likely to apply as mathematics teachers. All the teachers took part voluntarily in the study and they acknowledged that the questions in the interview were related to the concepts of the curriculum. All the interviews were conducted by us in Spanish. The interviewers endeavored to encourage the teachers to give full explanations of their understanding by nondirective questions such as "What do you mean by that?", "Could you explain that further?", "Do you want to say anything else about this question?"'

The interviews lasted about 20 min each. All interviews were transcribed and the transcripts subjected to the same analysis described above for the questionnaire. The excerpts showed in the Results section illustrate the successions of notions which characterize teachers' processes of situated cognition development through the described situation.

\section{EXPERIMENTAL DESIGN}

Based on the key concepts, we designed a questionnaire for students comprising three questions (Fig. 1) in which they were required to make predictions or explain experimental results related to absorption or emission spectra. The first two questions required the students to make a prediction about the emission or absorption of radiation by hydrogen atoms in well-defined conditions. This requires the use of a model in a qualitative manner, and excludes answers that refer to transitions between atomic levels in a merely generic manner. To respond correctly to Q1S and Q2S, students needed to be able to identify the states between which the atomic transition takes place and to relate them with the characteristics of the emitted or absorbed light. Figure 1 shows questions Q1S, Q2S, and Q3S of the questionnaire for students.

With the first question, Q1S (see Fig. 1), we inquire about the understanding of the absorption of radiation phenomenon. In it, $\mathrm{KC} 1, \mathrm{KC} 2$, and $\mathrm{KC} 3$ key concepts are assessed. A correct answer should state that atoms can only be found in stationary states $(\mathrm{KC} 1)$ and that the transitions between them are caused by the absorption of indivisible photons (KC2). In addition, the energy of a photon cannot be absorbed by more than one atom, nor can an atom absorb several photons in a single transition. Following this line of reasoning, an atom in the fundamental state will make a transition to a higher energy state if it absorbs a photon with an energy that is equal to the difference between the initial and final states (KC3). For question Q1S, photons of $10.2 \mathrm{eV}$ will be absorbed, producing a transition from the $-13.6 \mathrm{eV}$ state to the $-3.4 \mathrm{eV}$ state. Photons with any other energy may not be absorbed because it would move the atom to an energy state that does not correspond to any stationary state. For example, one students' correct standard answer reads as follows:

"A photon's energy is exactly equal to the energy difference between the fundamental state and the first excited state $\left(E_{1}=-3.4 \mathrm{eV}\right)$. The atom absorbs this energy and moves to the $E_{1}=-3.4 \mathrm{eV}$ state. In the second case, if the atom absorbed the photon energy, it would have an energy level of $-0.6 \mathrm{eV}$. However, there is no stationary state with that energy, and hence the atom cannot absorb this photon and move to another state." [physics $(P)$ student 1].

In question Q2S (see Fig. 1) we analyze whether students can predict the emission spectrum detected after hydrogen atoms are excited to a well-defined state. The correct answers should take into account that atoms in an excited state can make transitions only to states of lower energy ( $\mathrm{KC} 1)$. These transitions are random, i.e., they can be to any state of lower energy. When a single hydrogen atom makes a transition, it emits a single photon (KC3). For the case posed in the question, transitions can take place from -1.51 to $-3.4 \mathrm{eV}$ or from -1.51 to $-13.6 \mathrm{eV}$. In each one, a single photon is emitted. The atoms that have moved to the $-3.4 \mathrm{eV}$ state will later make a transition to the $-13.6 \mathrm{eV}$ state, emitting a second photon. In the emission spectrum, photons with three energies, and therefore three frequencies, will be detected. Three lines will appear, with an intensity that depends on the probability of each transition. Here is an example of a correct response: 
Q1S-Absorption spectra. The diagram on the right shows the energy of the hydrogen atom stationary states.

There are millions of hydrogen atoms in a vessel, all of which are in the lowest energy state, i. e. in the base state.

a) If we irradiate the vessel with photons that have $10.2 \mathrm{eV}$, what will happen to the gas atoms?

b) If we irradiate the vessel with photons that have $13 \mathrm{eV}$, what will happen to the gas atoms?

Q2S-Emission spectra. Hydrogen atoms contained in a vessel are excited by an electric discharge to the $-1.51 \mathrm{eV}$ stationary state (the diagram on the right shows the energy of the hydrogen atom stationary states). A spectroscope is then used to analyze the light emitted by the gas. What will be observed? (Choose one):

a) A single color line (light with a single frequency).

b) Several color lines (light with few frequencies).

c) A continuous spectrum, like a rainbow (light with all frequencies).

Support your answer.

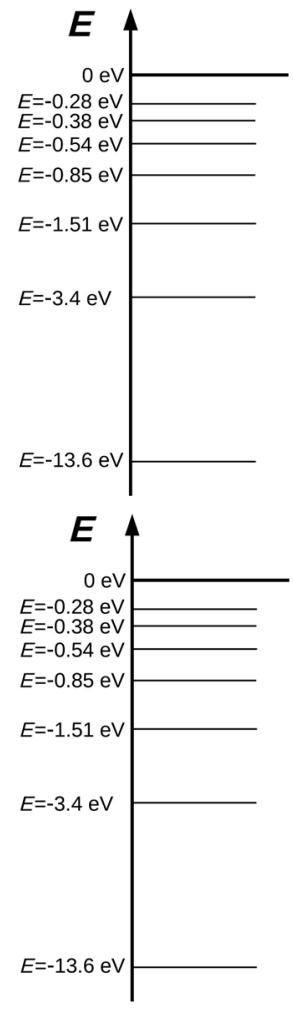

Q3S-Spectra lines intensity. How can you explain that there are some lines with higher intensity than others in a gas spectrum, as you can see in the picture of the Helium spectrum obtained in a high School laboratory?

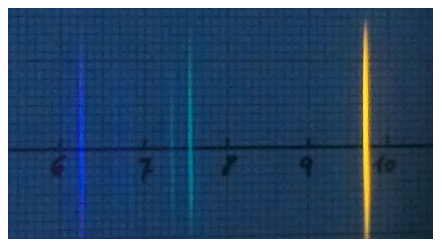

FIG. 1. English version of the questionnaire used to investigate students' ideas on absorption and emission spectra. Q1S-absorption spectra, prediction on absorption phenomena, Q2S-emission spectra, prediction on emission spectra, and Q3S-spectra lines intensity, interpretation of spectral lines' intensity.

"Some color lines (light with few frequencies) will be observed. We will observe permitted transitions $E_{2} \rightarrow$ $E_{1} \rightarrow E_{o}$ and $E_{2} \rightarrow E_{o}$." (P student 6$)$.

Question Q3S (see Fig. 1) asks about the interpretation of spectral lines' intensity and explores key concepts $\mathrm{KC} 2$ and $\mathrm{KC} 3$. A correct response to the question should involve matching a more intense line to a higher energy per unit of time of the amount of photons that have the frequency of the spectral line (KC2). As each photon has been generated in a random transition, a line of higher intensity also indicates that the transition which generates these photons is more likely to occur than others $(\mathrm{KC} 3)$. In the case considered, the most intense line is the yellow one (in the case of helium) and its frequency corresponds to the lowest photon energy, so the number of photons with that frequency emitted in a second should be higher than others.
This is equivalent to considering the atomic transition that originates the photons with the yellow frequency to be more probable than the others. A similar analysis will explain the other spectral lines intensities. These are some examples of students' correct standard answers to this question:

"The higher intensity indicates that more electrons have moved from one specific orbit to another." [high school (HS) student 6].

"The intensity difference is due to the transition probability; not all transitions are equally probable." (P student 18).

A different questionnaire was administered to the teachers in the course of the interviews (Fig. 2), with three questions that address essentially the same topics as the 
Q1T-Absorption spectra. The diagram shows the energy of the hydrogen atom stationary states. There are millions of hydrogen atoms in a vessel, all of them in the lower energy state, i. e. in the base state. We irradiate the vessel with photons that have energies between $1 \mathrm{eV}$ and $12.5 \mathrm{eV}$.

a) What will happen to the gas atoms?

b) What will we observe if we obtain the light spectrum after passing through the vessel?

Q2T-Emission spectra. Hydrogen atoms contained in a vessel are excited by an electric discharge to the $-1.51 \mathrm{eV}$ stationary state (the diagram shows the energy of the hydrogen atom stationary states). Then, a spectroscope is used to analyze the light emitted by the gas. What will be observed?

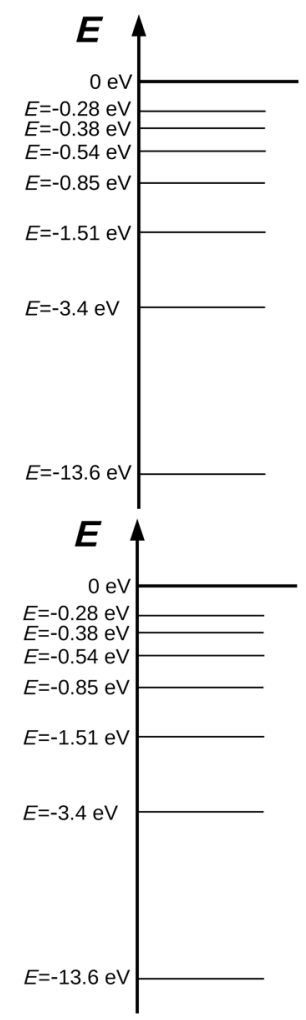

Q3T-Spectra lines intensity. How can you explain that there are some lines with higher intensity than others in a gas spectrum, as you can see in the picture of the Helium spectrum obtained in a high school laboratory?

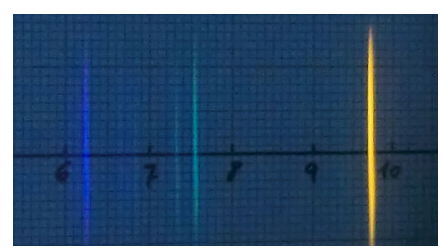

FIG. 2. English version of the interview questions for teachers used to investigate essentially the same topics as the questionnaire for students. In this case, the questions Q1T-absorption spectra and Q2T-emission spectra are more open ended than the first two questions of the questionnaire for students. Q1T prediction of absorption spectra, Q2T prediction on emission spectra, and Q3T-spectra lines intensity, interpretation of spectral lines' intensity.

questionnaire for students. The first two questions are more open ended than those presented to the students and allow us to analyze the explanations given or predictions made by teachers in greater detail. The third question is identical to the one presented to students. We used a different instrument for teachers and students because the oral interviews allow the interviewer to go into detail and request clarification of the answers given, where necessary.

Figure 2 shows the questions of the questionnaire for teachers. The first question Q1T (see Fig. 2) addresses the phenomenon of atomic absorption. In it we analyze key concepts $\mathrm{KC} 1, \mathrm{KC} 2$, and $\mathrm{KC} 3$. Correct answers must take into account that atoms can only be found in stationary states (KC1) and that transitions between them take place when indivisible photons are absorbed (KC2). An atom in the fundamental state will make a transition to a higher energy state if it absorbs a photon whose energy is equal to the energy difference between the initial and final states (KC3). In our case, only photons of $10.2 \mathrm{eV}$, (which will excite the atoms from the ground state to the $-3.4 \mathrm{eV}$ state) and photons of $12.09 \mathrm{eV}$ (which will excite the atoms from the ground state to the $-1.51 \mathrm{eV}$ state) can be absorbed. Unlike the question posed to the students, the incident radiation consists of photons that can be absorbed and photons that cannot, and this requires a more detailed explanation, which was rarely found among the written responses to the questionnaires. In addition, the presence of photons with different energies in the incident radiation makes teachers think in terms of the probability of successive transitions. However, and in accordance with KC3.4, hydrogen atoms that are in the states of -3.4 or $-1.51 \mathrm{eV}$ will emit radiation prior to absorbing another photon, because the successive absorption of photons would lead to the ionization of the atom, a phenomenon 
that is highly improbable and not observed in those conditions. For the (b) part of the question, a correct answer should take into account that two black lines will be observed on a continuous spectrum, corresponding to the 10.2 and $12.09 \mathrm{eV}$ photons absorbed by the gas. Explaining the absorption spectrum requires a greater level of consistency, and demands that one can relate parts (a) and (b) of the question. Here, the oral questionnaire allowed teachers to explain in detail, and, if necessary, allowed the interviewer to ask for clarifications.

The second question Q2T (see Fig. 2) is identical to the students' question Q2S, but the possible answers have been removed. The correct answers should take into account the key concepts $\mathrm{KC} 1$ and $\mathrm{KC} 3$, as Q2S does. We preferred the open-ended question to encourage teachers to explain their answer, rather than simply making a choice. In fact, this was the question originally intended for the students, but after the first tests it was observed that they gave incomplete answers, which were not possible to classify. For this reason, the multiple-choice format was added to the written questionnaire. The third question is the same as that posed to the students.

In the next section we present and analyze the results obtained and compare them with results reported in other research.

\section{RESULTS}

Table I presents the percentage of answers given to the questionnaire by the two groups of students. Table II presents the percentages of answers given in the interviews by the teachers. We have marked with a $(\cdots)$ the key concepts that are not assessed in each question. Correct answers have been categorized as category A, while category $\mathrm{B}$ comprises the ties in relation to the key concepts. We also found some incorrect answers not clearly related to any key concept. In many cases these lacked internal logic. We have classified them in category $\mathrm{C}$ as "unclassifiable." In all of the tables, the percentages were rounded to the nearest $0.5 \%$, since a difference between $0.2 \%$ and $0.4 \%$ in sample sizes of $37 \mathrm{HS}$ students or $34 \mathrm{P}$ students represents much less than one person.
The results show that fewer than $10 \%$ of high school students were able to predict or interpret the emission and absorption spectra results. In the case of the physics undergraduate students, we found a notable progression in the learning of the emission phenomena (over $50 \%$ of correct answers in Q2 and Q3) but the result was significantly lower for the absorption phenomenon (only $23 \%$ gave a correct answer). Taking into account that physics students have done specific quantum mechanics courses, these results can be considered poor. We have to highlight a high percentage of unanswered questions among high school students. This result is similar to that obtained in a study of this subject with high school students in Spain [53]. Moreover, our sample of HS students showed a higher percentage of incoherent or unclassifiable responses, between $15 \%$ and $30 \%$.

Regarding teachers (see Table II), only $39.5 \%$ were able to account for the frequency or intensity of emission spectral lines (category A, Q2 and Q3, respectively) and fewer than $30 \%$ could make predictions about the absorption process (category A, Q1). There is also a significant percentage of "no answer" (between 16.5\% and 43\%). This result shows that the concepts and models involved in the phenomena covered in the interviews were complex for the teachers.

\section{A. Students' difficulties regarding the emission and absorption phenomena}

Table I shows that some HS and P students' explanations reveal difficulties with energy quantization in the atom (KC1) when explaining the absorption or emission of energy (category B.1). As shown below, two main types of alternative explanation were detected.

One explanation states that the atom can have a different energy than that of the stationary states. This misunderstanding is a direct violation of energy quantization (KC1); we found this reasoning in $12.0 \%$ of physics students and $5.0 \%$ of high school students when explaining absorption (Q1). In this "alternative" explanation, students believed that photons are always absorbed and the atoms acquire an energy that does not correspond to any stationary state:

TABLE I. Percentage of answers given to the questions by 37 high school Students (HS) and 34 Physics Students (P).

\begin{tabular}{|c|c|c|c|c|c|c|}
\hline \multirow[b]{2}{*}{ Category } & \multicolumn{2}{|c|}{ Question Q1: absorption } & \multicolumn{2}{|c|}{ Question Q2: emission } & \multicolumn{2}{|c|}{ Question Q3: intensity } \\
\hline & HS & $\mathrm{P}$ & HS & $\mathrm{P}$ & HS & $\mathrm{P}$ \\
\hline A. Emission or absorption are correctly explained. & 0 & 23.0 & 5.5 & 67.0 & 8.0 & 50.0 \\
\hline B.1. Have difficulties with atom quantization & 16.0 & 12.0 & 2.5 & 3.0 & $\cdots$ & $\ldots$ \\
\hline B.2. Have difficulties with radiation quantization & 2.5 & 12.0 & $\cdots$ & $\cdots$ & 13.5 & 3.0 \\
\hline $\begin{array}{l}\text { B.3. Have difficulties with the interaction } \\
\text { between an atom and a photon. }\end{array}$ & 41.0 & 50.0 & 40.5 & 15.0 & 27.0 & 26.0 \\
\hline C. Lack of coherence or not classifiable & 13.5 & 0 & 19.0 & 9.0 & 30.0 & 9.0 \\
\hline Did not answer & 27.0 & 3.0 & 32.5 & 6.0 & 21.5 & 12.0 \\
\hline
\end{tabular}


TABLE II. Percentage of answers given to the questions by 30 teachers (T). The question Q1 for teachers has two parts concerning the atom (part a: What will happen to the gas atoms?) and concerning the radiation (part b: What will we observe if we obtain the light spectrum?).

\begin{tabular}{|c|c|c|c|c|}
\hline \multirow[b]{2}{*}{ Category } & \multicolumn{2}{|c|}{ Question Q1: absorption } & \multirow{2}{*}{$\begin{array}{l}\begin{array}{c}\text { Question Q2: } \\
\text { emission }\end{array} \\
\mathrm{T}\end{array}$} & \multirow{2}{*}{$\begin{array}{c}\begin{array}{c}\text { Question Q3 } \\
\text { intensity }\end{array} \\
\mathrm{T}\end{array}$} \\
\hline & $\mathrm{T}(\mathrm{a})$ & $\mathrm{T}(\mathrm{b})$ & & \\
\hline A. Emission or absorption are correctly explained. & 27.0 & 10.0 & 39.5 & 39.5 \\
\hline B.1. Have difficulties with atom quantization & 23.5 & 10.0 & 13.5 & $\ldots$ \\
\hline B.2. Have difficulties with radiation quantization & 0 & 0 & $\ldots$ & 13.5 \\
\hline $\begin{array}{l}\text { B.3. Have difficulties with the interaction } \\
\text { between an atom and a photon. }\end{array}$ & 26.5 & 23.5 & 13.5 & 23.5 \\
\hline C. Lack of coherence or not classifiable & 6.5 & 13.5 & 10.0 & 0 \\
\hline Did not answer & 16.5 & 43.0 & 23.5 & 23.5 \\
\hline
\end{tabular}

"By illuminating with $10.2 \mathrm{eV}$ photons the atoms will rise to an external energy level (with more energy) that corresponds to $\Delta E=10.2 \mathrm{eV}$

By illuminating with $13 \mathrm{eV}$ photons the same will occur" (HS student 16, question Q1).

"By illuminating with $10.2 \mathrm{eV}$ photons the atoms will absorb all this energy and pass to the state of $-3.4 \mathrm{eV}$.

By illuminating with $13 \mathrm{eV}$ photons the atoms will have an absorption energy of $0.6 \mathrm{eV}$." (P student 19, question Q1).

The second kind of misunderstanding is based on the belief that the $0 \mathrm{eV}$ is the lowest energy state (the base state). Because this is not strictly a quantum misconception, it is not included among our key concepts. In this alternative explanation, also found by Ivanjek et al. [54], students do not know how to interpret the energy sign of a system of particles and they think that the base state (lowest energy) always has an energy level of $0 \mathrm{eV}$. This line of reasoning is found in both the first question Q1 (11.0\% HS students) and the second question Q2 ( $2.5 \%$ of HS students and $3.0 \%$ of P students). The next ones are some examples from the students' questionnaire:

"By illuminating with $10.2 \mathrm{eV}$ photons, an electron will leap to the state just under that amount of energy, to the penultimate state.

By illuminating with $13 \mathrm{eV}$ photons, the same will occur as in the previous case." (HS student 3, Q1).

"By illuminating with $10.2 \mathrm{eV}$ photons, electrons will move to the highest energy level reachable with that energy, to the $-3.4 \mathrm{eV}$ state.

By illuminating with $13 \mathrm{eV}$ photons, electrons will reach the same energy level as in the previous case, because photons do not have the $13.6 \mathrm{eV}$ frequency." (HS student 15, Q1).

"Several colored lines will be observed in the emission spectrum. We will observe the photons emitted in the transition to the lowest energy states. We will see only a few frequencies because there are only five energy levels below this one" (P student 10, Q2).

Regarding students' explanation on the quantum radiation, Table I (category B.2) shows that a fraction of student's responses reveal difficulties using the quantum radiation model based on the photon concept (KC2) to explain the absorption (Q1) and the intensity of spectral lines (Q3). As we show below, we have identified two main difficulties in this category.

A first alternative explanation states that a photon can be partially absorbed. This explanation is given solely in the case of absorption (Q1) by $2.5 \%$ of HS students and by $12.0 \%$ of P students. It is a clear violation of the energy quantization of radiation, according to which photons are indivisible (KC2.1). The reasoning behind this explanation is based on the belief that atoms always absorb photons and, thereafter, a stationary state must be reached. If the photon energy is greater than the energy needed to make a transition, its energy is partially absorbed, breaking the quantum radiation principle according to which photons are indivisible energy quanta. The following responses are some examples:

"By illuminating with $13 \mathrm{eV}$ photons, the atoms will absorb the photons and will pass to a higher energy state, that of $-0.85 \mathrm{eV}$. The excess energy is not absorbed as it is not required to reach the higher state." (HS student 27, Q1).

"When illuminating with $13 \mathrm{eV}$ photons, excitations will occur in which the atoms will be distributed in the possible energy levels emitting the excess energy, if it exists, as light." (P student 16, Q1).

The second difficulty identified involves arguments that do not relate the radiation intensity with the number and the energy of photons, but instead attribute a higher intensity to the radiation that is formed by higher energy photons. This difficulty, which fails to take key concept KC2.3 into account, was only detected in Q3, as it is the only question that 
demands an explanation of the radiation intensity. This alternative explanation was found in $13.5 \%$ of HS students and in 3.0\% of $\mathrm{P}$ students. The following responses are some examples:

"The line intensity is greater because the jump made by the electron from one orbit to another is greater." (HS student 25, Q3).

"The line intensity is higher because the frequency is greater." (HS student 29, Q3).

Regarding students' explanations on the interactions between an atom and a photon, Table I (category B.3) shows that some students have difficulties when explaining this interaction in a scientific way (key concept KC3). We have detected four major types of alternative explanations. Below, we discuss each one and state the percentage of students that reported them.

The first alternative explanation includes a set of arguments in which it is postulated that there are no transitions between stationary states (contrary to KC3.1). Thus, it is concluded that the energy diagram represents the energy of stationary states and, simultaneously, the energy of the emitted or absorbed photons. This misconception is found in the three questions: when explaining the absorption of radiation in Q1 (11.0\% of HS students and $14.5 \%$ of $\mathrm{P}$ students), when explaining the emission in Q2 (11.0\% of HS students and $9.0 \%$ of $\mathrm{P}$ students) and when explaining the intensity of the spectral lines in Q3 (8.0\% of HS students and $9.0 \%$ of $\mathrm{P}$ students). When explaining the lines' intensity, a higher intensity of radiation is related to the presence of more electrons in a certain stationary state or the higher intensity is attributed to the radiation emitted from the higher energy level. The following are some examples of this reasoning:

"When illuminating with $10.2 \mathrm{eV}$ photons, such photons do not have enough energy to excite the hydrogen atoms' electrons, so nothing will happen.

When illuminating with $13 \mathrm{eV}$ photons, [nothing happens as] they don't have the energy required to excite the atoms either; the threshold is $13.6 \mathrm{eV}$." (P student 17, Q1).

"A monochromatic single line will be seen because, for stationary states, a well-defined energy corresponds to a single frequency." (P student 20, Q2).

"There are lines with higher intensity in the spectrum because they correspond to higher energy levels, which emit more intensely." (P student 15, Q3).

There are explanations that do not even take into account the state of the atom and suppose that it absorbs the photons that have the same energy as the stationary states represented in the diagram (in absolute terms) and, in some cases, a near value. This alternative view has also been found by Zollman et al. and Ivanjek et al. [55,56]. The following response is an example of this:

"By illuminating with $10.2 \mathrm{eV}$ photons nothing will happen to the atoms, because that energy is very distant from the atom energy states.

By illuminating with $13 \mathrm{eV}$ photons, since this energy is close to one of the hydrogen stationary states, the atoms will undergo a change." (HS student 24, Q1).

The second alternative explanation relates the radiation emitted by the atom with only one of the states between which the transition takes place. We have identified this misunderstanding only in the emission of radiation, in both Q2, when asking about the number of spectral lines observed in the emission spectrum (16\% of HS students), and Q3, when asking about the spectral lines' intensity (11.0\% of HS students and $12.0 \%$ of P students). In all cases, the explanations fail to consider the two states between which the atomic transition takes places to emit the photon. They only consider the energy state from which the electron leaves or the state where it ends up. The following are some examples of these responses:

"It only emits one line, because by jumping from one level it provides the light equivalent to this level." (HS student 5, Q2).

"If there are lines more intense than others it means that it is emitting more radiation due to some states than to others. This is because the transition probability to certain states is greater than the transition probability to other states. The higher the probability, the more transitions, and the more radiation with a frequency corresponding to that state." (P student 1, Q3).

The third alternative explanation claims that the transitions to lower energy states take place directly to the ground state, contrary to key concept KC3.2. This would imply that the number of spectral lines observed in the emission spectra would be much lower than that experimentally observed. This misunderstanding, which has also been identified by Ivanjek et al. [57], was found in both Q2, when asking about the lines observed in the emission spectrum (11.0\% of HS students and $6.0 \%$ of P students) and Q3, when asking about the intensity of spectral lines (3.0\% of P students). The following responses are some examples of this:

"A line of one color is emitted. We will observe the light corresponding to the wavelength used to produce that transition, because when the effect was produced, it was absorbed by the atom, and now, when the energy stimulation stops, it emits that frequency and returns to its stable state." (HS student 30, Q2). 
"There are more intense spectral lines than others due to the transition probability between some states to the ground state." (P student 8, Q3).

A variant of this misunderstanding attempts to account for more spectral lines by claiming that the electron emits a photon every time "it passes through" a stationary state in its transition to the ground state. An example is the following answer:

"Some colored lines will be observed, each one corresponding to the stationary states overtaken by the electron." (HS student 29, Q2).

The fourth alternative explanation postulates that the conservation of energy is not applied in the interaction process between radiation and matter. It is not strictly a quantum-based line of reasoning but rather a consequence of a misunderstanding of the key concepts, apparently due to thinking that the atom must always undergo a transition to a higher energy state, regardless of the photons' energy. This reasoning is only found in relation to the absorption phenomena (Q1), but $30.0 \%$ of HS students and $35.5 \%$ of $\mathrm{P}$ students subscribed to this view. The following are some examples of these:

"By illuminating with $10.2 \mathrm{eV}$ photons the atoms will have the energy to reach the second level $(E=-3.4 \mathrm{eV})$.

When illuminating with $13 \mathrm{eV}$ photons the atoms will get to the fifth energy level $(E=-0.54 \mathrm{eV})$." (HS student 23).

"By illuminating with $10.2 \mathrm{eV}$ photons, the atoms will make a transition to the $-3.4 \mathrm{eV}$ state.

By illuminating with $13 \mathrm{eV}$ photons, the atoms will get to the $E=-0.85 \mathrm{eV}$ energy state, as they do not have enough energy to reach the $E=-0.54 \mathrm{eV}$ energy state." (P student 13).

A combined analysis of the answers to the three questions reveals that not a single high school student answered the three questions correctly, and there was not even anyone who correctly answered Q2 and Q3 (concerning the emission of radiation). This result shows that high school students are far from having acquired a quantum model to explain the emission and absorption of radiation. With regard to physics students, only $20.5 \%$ answered the three questions correctly, although the percentage rises to $47.1 \%$ for Q2 and Q3. These results suggest that physics students do not interpret spectra with a consistent model but answer each question separately in an ad hoc fashion, falling back on alternative conceptions when faced with less familiar questions.

\section{B. Teachers' difficulties regarding the emission and absorption phenomena}

Regarding teachers, as shown in Table II (category B.1), we find that they have difficulty with the quantum model of the atom. However, the difficulty encountered does not relate specifically to the quantization of energy, but rather to the assumption that the lower energy state is $0 \mathrm{eV}$. When asked about absorption, in Q1, 23.5\% of the teachers exhibited this type of reasoning and consider that an atom makes a transition to a lower energy state when a photon is absorbed, although the atom gains energy. When asked about emission, in Q2, 13.5\% of the teachers show this type of reasoning and consider that an atom makes a transition to a higher energy state when a photon is emitted, although the atom loses energy. This is an extract from one teacher's interview:

"Teacher, (degree in chemistry, 20 years teaching experience, Q1): The electrons would move to a higher energy state.

Interviewer: Could you say to which one?

$T_{9}$ : To which one? It would be necessary to... [silence].

I: Radiation has photons between 1 and $12.5 \mathrm{eV}$.

$T_{9}$ : Between 1 and $12.5 \mathrm{eV}$ ? Between 1 and $12.5 \mathrm{eV}$, all the spectrum?

I: The whole spectrum from 1 to $12.5 \mathrm{eV}$. I mean, you'd have $1.5 \mathrm{eV}$ photons and all intermediate values.

$T_{9}$ : They would reach all states, except the lowest one [that of $-13.6 \mathrm{eV}$ ].”

Table II shows that $13.5 \%$ of teachers have difficulty applying the quantum radiation model (category B.2), specifically to explain the intensity of spectral lines. All of them fall back on the same alternative conception, concerning $\mathrm{KC} 3.2$, which is that the most intense radiation is that formed by the photons with the highest energy, without considering the number of photons involved. This reasoning is equivalent to considering that all the transitions in the atom have the same probability of occurring, so the higher intensity line corresponds to the higher energy shift between levels. Here is an example of a response from a teacher:

"The line intensity is greater because there is a higher energy shift between levels. There is a different distance between each one, it is a consequence of that, there is a higher energy shift." (teacher 17, degree in chemistry, 20 years teaching experience, Q3).

We note that we have not found evidence of difficulties concerning key concept KC2.2 (relating the energy of each photon to the radiation frequency) in teachers' or students' 
responses. This may be because the questions did not ask for both magnitudes to be explicitly related. In future studies, we should include questions that directly address this key concept to find out whether it is part of the teachers' and students' models.

Table II shows that a number of teachers have difficulties with the interaction between an atom and a photon (category B.3). The difficulties in this category, related to $\mathrm{KC} 3$, affect all of the questions posed and are divided into five main types of alternative explanations. Of these, the first four coincide with the ones identified among the students in category B3.

The first alternative explanation is based on the proposition that there are no transitions between stationary states when an atom emits or absorbs radiation. We identify this when we ask about the absorption in Q1 $(13.5 \%$ of teachers), when we ask about emission in Q2 (10.0\% of teachers), and when asking about the intensity of the spectral lines in Q3 (13.5\% of teachers). The following are examples of responses given by the teachers:

"The line intensity is higher because there are more electrons in the corresponding energy level." (teacher 16, degree in physics, 10 years' teaching experience, Q3).

"Teacher ${ }_{10}$ : The electrons that have an energy level greater than $12.5 \mathrm{eV}$ can interact and leave the atom.

Interviewer: Taking into account that photons have energies between 1 and $12.5 \mathrm{eV}$ ?

$T_{10}$ : Yes, because here we have $-13.6,-3.4,1.51 \mathrm{eV} \ldots$

I: All the electrons are in the base state before illuminating.

$T_{10}$ : Yes. Then, Yes.

I: And, what will we observe if we obtain the light spectrum after passing through the gas?

$T_{10}$ : I guess we will observe the absorbed radiation spectrum. A line of $3.4 \mathrm{eV}$, all the lines represented in the diagram. I do not understand why. For example, the 13.6 and $0.28 \mathrm{eV}$ could not have been absorbed." (teacher 10, degree in chemistry, 23 years teaching experience, Q1).

As can be seen in teacher 10's response, there are also teachers who think that the photons whose energy coincides with the atom's stationary states are the ones that can be absorbed, whether or not they are occupied by electrons.

The second alternative explanation claims that, when undergoing a transition, the intensity of the light emitted depends solely on one of the states between which the transition takes place. Teachers ascribe to this conception only when explaining the intensity of the spectral lines, attributing greater intensity to the transitions that take place to the stationary states with higher energy, in Q3 (10.0\% of teachers).

A smaller percentage of teachers ascribe to alternative explanations three and four. $3.5 \%$ of teachers (only one teacher) gave the third alternative explanation and said that the transitions take place directly to the fundamental state. This response was identified in Q2, when asking about the lines that are observed in the emission spectrum. With regard to the fourth alternative explanation, this was found only in Q1 and 6.5\% of the teachers ascribed to this. These teachers believe that the atom always absorbs photons from radiation and undergoes transitions to some excited state, although this means that the principle of energy conservation is violated.

The fifth alternative explanation claims that all the atoms will absorb many photons and make successive transitions, first from the ground state to an excited state and then from the reached state to a higher-energy state, and so on, up to ionize the atom. Although we have mentioned that transitions between excited states are possible but highly improbable, this explanation makes no reference to the low transition probability; on the contrary, it claims that the transitions occur whenever the photon energy matches the energy difference between excited states. This difficulty was detected only in relation to question Q1, and only 6.5\% of teachers encountered it. The following response is an example:

"Teacher 6 : If you irradiate the gas with photons whose energies are between 1 and $12.5 \mathrm{eV}$, the $12.5 \mathrm{eV}$ photons will do nothing. But the photons with $10.2 \mathrm{eV}$ will make an electron transition from the -13.6 state to $-3.4 \mathrm{eV}$ state. The same will happen with all the photons that match the difference between these levels. When the atom is at $-3.4 \mathrm{eV}$ and a $3.5 \mathrm{eV}$ photon arrives, it will move from that level to ionize. Or something like that. I do not know how many possibilities there are." (teacher 6, degree in chemistry, 30 years teaching experience, Q1).

While students did not encounter this difficulty it does not mean, in our view, that they had overcome this misconception. The question Q1S asked the students what would happen to the atom when it absorbs radiation, but did not explicitly outline the possibility of successive transitions, so students may not have thought about it or they may have ignored it in their responses.

When we make a combined analysis of the three questions, we find that only $10 \%$ of teachers answered them all correctly and only $23.5 \%$ of them answered questions Q2 and Q3 correctly (the questions concerning to the emission phenomena), although $39.5 \%$ of responses were correct for each of these topics. The fact that up to $16 \%$ of teachers who resorted to an alternative conception for one of them did not do it for the other one suggests that 
teachers interpreted each situation separately, without reasoning using a coherent model.

\section{DISCUSSION}

The results show that students and teachers experience difficulties in comprehending the same key concepts. In the case of the students, we observe a learning progression but the same comprehension difficulties persist.

With regard to the difficulties concerning the quantization of energy in the atom, the results (Table I, category B.1) show that the $P$ students have a better comprehension of the atom energy diagram than the HS students, as well as a clearer understanding of the concept of energy. However, a larger percentage of $\mathrm{P}$ students believe that an absorption must take place if atoms are irradiated even if the atom does not acquire the energy of a steady state. These alternative explanation suggest that the physics students, although having a better comprehension, are mixing together classical and quantum concepts with regard to atoms, as has been found in previous research [58,59]. Moreover, in the results of interviews with teachers, it is shown that up to $23.5 \%$ of the subjects had difficulties using the quantum model of the atom (Table II, category B.1), although in no case did they violate the energy quantization principle. This suggests that they have an "iconic" conception of the atom, a static representation that includes the quantization of energy without any functional capacity, and that the difficulties arise when they attempt to use the quantum model to explain or predict radiation emission and absorption phenomena.

In relation to the difficulties of comprehending the quantum model of radiation, our results indicate that the P students have a better understanding of this model than the HS students do (Table I, category B.2). However, the idea that a transition takes place whenever an atom is subjected to radiation, even though this violates the energy quantization principle, is still prevalent among the $\mathrm{P}$ students. The explanations given by the HS students and the P students suggest that they conceive of photons as classical particles that can transfer any amount of energy in an interaction with other particles. This result is consistent with other results obtained in previous research that highlight this same type of difficulty [60,61], although in our study we refer to these difficulties in relation to atomic spectra. Moreover, that misconception has also been found in high school physics textbooks, which consider that an electron can absorb part of the energy of a photon when they interact in the Compton effect [62]. Conversely, the teachers' responses in the interviews show that they understand that radiation is composed of photons, understood as indivisible quanta (KC2.1), but they are not able to use the quantum model of radiation to explain all of the characteristics of spectral lines. Indeed, they have problems when they try to interpret the intensity of the radiation (Table II, category B.2, question Q3).

Regarding difficulties on the interactions between an atom and a photon (Table I, category B.3), we observe that the percentage of $\mathrm{P}$ students that resort to alternative explanations to respond to the question about radiation absorption (Q1) is 9\% higher than for the HS students. Further, both groups use the same alternative explanations to respond to this question: either they think that there are no transitions between stationary states during the absorption (11\% HS students and $14.5 \%$ P students) or they think that photons are absorbed to produce transitions even though this would violate the law of conservation of energy (30\% HS students and $35.5 \% \mathrm{P}$ students). The percentage of $\mathrm{P}$ students who resort to alternative explanations when responding to the question on the emission spectrum $(\mathrm{Q} 2)$ is in every case smaller than that of the HS. Table I also shows that the percentage of $\mathrm{P}$ students that responded correctly to this question was much greater than the percentage of HS students. This is what one would expect given the difference in the number of years of study of physics by the two groups. However, the percentage of $\mathrm{P}$ students that resort to alternative explanations when interpreting the intensity of the spectral lines is practically the same. The time that the $\mathrm{P}$ students have spent studying physics has not enabled them to overcome the difficulties that the HS students have already encountered. This indicates that they have not achieved an in-depth understanding of quantum models during their university-level studies, which is why the difficulties persist when they try to explain aspects related to the interaction between radiation and atoms. The results of the interviews with the teachers support the conclusion that their difficulties have to do principally with explaining the processes of emission and absorption of radiation (Table II, category B.3). In fact, we have not thus far found any difficulties in relation to the quantization of energy in the atom or in radiation among this group.

For all of the groups of subjects, most alternative conceptions were found in relation to the emission and absorption process. This result was predictable because a process model is necessary to interpret or explain those phenomena. Process models do not account for a physical entity but for a phenomenon or change. In turn, models are needed that account for the physical entities involved in that phenomenon (atoms and light, in our case). It is not surprising, therefore, that these models stand out on account of their difficulty [63].

\section{CONCLUSIONS AND IMPLICATIONS FOR FUTURE WORK}

When drawing conclusions and implications for teaching, it is necessary to bear in mind that the questionnaire and interviews were carried out with a small number of 
students and teachers at Spanish high schools and universities. Thus, we cannot produce evidence for more general contexts. Our study has not been designed to present conclusive evidence on all high school students' and teachers' difficulties with learning about atomic spectra and the interaction between atoms and radiation; in fact, there may be difficulties due to other misconceptions not explicitly taken into account in the key concepts of this study. However, we have found that the results obtained in this study are consistent with results found in other studies carried out with student samples from other countries $[64,65]$.

As we commented in Sec. IV, the Spanish national high school curriculum states that students must be able to "explain, using the laws of quantum physics, a series of experiences that classical physics is unable to explain, such as discontinuous spectra [...]." The key concepts presented in Sec. II of this paper are essential to establish any quantum model that permits one to explain the emission and absorption of radiation by atoms and in particular, gas spectra, as required by the national curriculum. Nevertheless, the results obtained show that high school students have a very low level of understanding of the quantum model of emission and absorption of radiation, with no students able to answer more than one question correctly. The final-year physics students admittedly achieved somewhat better results; but fewer than $50 \%$ were able to answer more than one question correctly. Furthermore, the data from the questionnaire show that a significant number of students proposed explanations that were limited to describing the phenomena or that use remembered concepts without any logical consistency. These data are coherent with the results from cognitive science on reasoning complexity [66]. We conclude that students' ability to handle complex reasoning should be upgraded instead of merely describing phenomena to linking these phenomena to the interpretative concepts and laws used in the scientific model studied in relation to spectra phenomena.

The data also show that these difficulties not only arise in relation to the interaction process between atoms and radiation. They may be caused by misconceptions about the atom or about the quantum nature of radiation, so they may already be present when spectra are studied and have remained latent, reemerging when other atomic or radiative phenomena are studied. Indeed, some difficulties are not even unique to quantum physics, such as the mistaken belief that the value of the minimum energy for the electron in an atom is zero (and cannot be negative) or violations of the principle of energy conservation. The results show that all these aspects must be taken into account to ensure proper understanding of the quantum emission and absorption of radiation.

This study provides evidence that difficulties are manifested at all levels, providing further evidence of their persistence. In addition, the high school teachers in our study did not appear to possess a model of the emission and absorption of radiation capable of explaining atomic spectra. Fewer than $25 \%$ of them were able to explain the frequencies and intensities of spectral lines in emission phenomena, a percentage that decreases to $10 \%$ when absorption is included. The results show that as high school teachers have a poor level of understanding, they can hardly facilitate effective learning to help high school students to overcome their difficulties. This may be due to poor teacher training in modern physics, although all of the teachers interviewed have received training on content and pedagogy for a year before becoming practicing teachers and, furthermore, have over 10 years' teaching experience. It would require them to take part in relevant training activities aimed at improving both their subject content knowledge and their pedagogical content knowledge.

Gas spectra are a phenomenon that historically led to the introduction of the quantum hypothesis in the atom. Academically, the emission spectra are used to introduce the Bohr atom, which is later used to study more advanced quantum atomic models. But it is done in a cursory way; no opportunities are given to the students for using the model to explain some other emission or absorption phenomena, including the absorption spectra. Our research results question the effectiveness of this teaching approach. In fact, we find that the majority of teachers and students are unable to predict how atoms interact with radiation. Teaching by lectures would not appear to the be the most appropriate manner to achieve the goal set in the curriculum, which is to "explain, using the laws of quantum physics, a series of experiences that classical physics is unable to explain, such as discontinuous spectra." First, chalk and talk teaching of the concepts does not usually provide opportunities for the students to participate in the construction of models and to use them in diverse situations, and this makes it more difficult for students to learn them. Second, the limited ability of teachers to use the quantum model of emission and absorption of radiation means the teaching is less effective and this contributes to the difficulties instead of helping to overcome them.

Our study also provides further evidence about the characteristics (key concepts) and the complexity of a sound understanding of the quantum absorption and emission of radiation, which is central in introductory and advanced courses in quantum physics. Moreover, the diversity of the difficulties encountered (category B, Table I) highlights the complexity of the quantum model of emission and absorption of radiation, the acquisition of which provides students with the opportunity to recognize the conflicts and fundamental issues that led to the emergence of quantum physics.

We are currently involved in designing a teachinglearning sequence (TLS) for quantum physics foundation courses to help teachers and students to understand and 
use Bohr's model and the quantum radiation model based on the photon concept. In view of the learning difficulties found in this study, the TLS takes into account that the introduction of both models and their use to explain the spectra should reveal the profound differences between the classical and the quantum models for the interaction between radiation and matter. When designing future materials, we will set out to highlight the difficulties that the classical model could not overcome, and how such difficulties led to the establishment of the first model that includes the quantization of energy. We have obtained some initial results of the TLS implementation that indicate a positive impact on students' understanding. However, more work is needed to increase the sample of students and the number of years of implementation.
[1] J. M. Sánchez Ron, Historia de la Física Cuántica (Crítica, Barcelona, 2001), p. 15.

[2] A. B. Arons, Teaching Introductory Physics (John Wiley \& Sons, New York. 1997), p. 266.

[3] P. Fletcher and I. Johnston, Quantum mechanics: exploring conceptual change, Proceedings of the Annual Meeting of the National Association for Research Science Teaching (1999), p. 28, http://www.physics.umd .edu/perg/qm/qmcourse/NewModel/research/qm_narst_ full.pdf.

[4] S. B. McKagan, K. K. Perkins, M. Dubson, C. Malley, D. Reid, R. Lemaster, and C.E. Wieman, Developing and researching $\mathrm{PhET}$ simulations for teaching quantum mechanics, Am. J. Phys. 76, 406 (2008).

[5] G. Pospiech, Teaching the EPR paradox at high school?, Phys. Educ. 34, 311 (1999).

[6] G. Pospiech, Uncertainty and complementarity: the heart of quantum physics, Phys. Educ. 35, 393 (2000).

[7] M. Michelini, R. Ragazzon, L. Santi, and A. Stefanel, Proposal for quantum physics in secondary school, Phys. Educ. 35, 406 (2000).

[8] D. Hoekzema, E. Van Den Berg, G. Schooten, and L. Van Dij, The particle/wave-in-a-box model in Dutch secondary schools, Phys. Educ. 42, 391 (2007).

[9] G. Kalkanis, P. Hadzidaki, and D. Stavrou, An instructional model for a radical conceptual change towards quantum mechanics concepts, Sci. Educ. 87, 257 (2003).

[10] P. Hadzidaki, The Heisenberg microscope: a powerful instructional tool for promoting meta-cognnitive and meta-scientific thinking on quantum mechanics and the "nature of science", Sci. Educ. 17, 613 (2008).

[11] O. Levrini and P. Fantini, Encountering productive forms of complexity in learning modern Physics, Sci. Educ. 22, 1895 (2013).

[12] C. Baily and N. D. Finkelstein, Development of quantum perspectives in modern Physics, Phys. Rev. ST Phys. Educ. Res. 5, 010106 (2009).

[13] C. Baily and N. D. Finkelstein, Teaching and understanding of quantum interpretations in modern physics courses, Phys. Rev. ST Phys. Educ. Res. 6, 010101 (2010).

[14] C. Baily and N. D. Findelstein, Refined characterization of student perspectives on quantum physics, Phys. Rev. ST Phys. Educ. Res. 6, 020113 (2010).
[15] C. Baily and N. D. Findelstein, Teaching quantum interpretations: Revisiting the goals and practices of introductory quantum physics courses, Phys. Rev. ST Phys. Educ. Res. 11, 020124 (2015).

[16] H. Fischler and M. Lichfeld, Modern Physics and students' conceptions, Int. J. Sci. Educ. 14, 181 (1992).

[17] R. Justi and J. Gilbert, History and philosophy of science through models: some challenges in the case of 'the atom', Int. J. Sci. Educ. 22, 993 (2000).

[18] K. S. Taber, When the analogy breaks down: modelling the atom on the solar system, Phys. Educ. 36, 222 (2001).

[19] See Ref. [4].

[20] D. Gil and J. Solbes, The introduction of modern Physics: overcoming a deformed vision of science, Int. J. Sci. Educ. 15, 255 (1993).

[21] C. Singh, Students understanding of quantum mechanism, Am. J. Phys. 69, 885 (2001).

[22] K. Zuza, J. M. Almudí, A. Leniz, and J. Guisasola, Addressing students' difficulties with Faraday's law: A guided problem solving approach, Phys. Rev. ST Phys. Educ. Res. 10, 010122 (2014).

[23] J. Osuna García, J. Martínez-Torregrosa, J. Carrascosa Alís, and R. Verdú Carbonell, Planificando la enseñanza problematizada: el ejemplo de la óptica geométrica en educación secundaria, Enseñanza de las Ciencias 25, 277 (2007).

[24] C. Becerra-Labra, A. Gras-Martí, and J. MartínezTorregrosa, Effects of a problem-based structure of Physics contents on conceptual learning and the ability to solve problems, Int. J. Sci. Educ. 34, 1235 (2012).

[25] A. Einstein and L. Infeld, The Evolution of Physics (Cambridge University Press, Cambridge, England, 1938), https://archive.org/details/evolutionofphysi033254mbp.

[26] J. L. Domènech Blanco, J. Martínez Torregrosa, and F. Savall Alemany, ¿Los libros de texto de bachillerato introducen adecuadamente los modelos atómicos de Thomson y Rutherford?, Enseñanza de las Ciencias 31, 29 (2013).

[27] N. Bohr, On the constitution of atoms and molecules, Philos. Mag. 26, 1 (1913).

[28] M. Jammer, The Conceptual Development of Quantum Mechanics (Mc Graw Hill, New York, 1966), p. $30-37$. 
[29] L. Navarro, Einstein y los comienzos de la física cuántica: de la osadía al desencanto, Investigación y Ciencia 338, 38 (2004).

[30] F. Savall Alemany, J. L. Domènech Blanco, and J. Martínez Torregrosa, La introducción del concepto de fotón en bachillerato, Revista Brasileira de Ensino de Física 35, 2404 (2013).

[31] F. J. Rutherford, G. Holton, and F. G. Watson, Project Physics (Holt, Rinehart and Winston, California, 1970).

[32] H. Fischler, Introduction to quantum physics-development and evaluation of a new course, Proceedings of the Annual Meeting of the National Association for Research and Science Teaching (1999), p. 32, http://perg.phys.ksu.edu/ papers/narst/QM_papers.pdf.

[33] D. Lindley, Incertidumbre (Ariel, Barcelona, 2008).

[34] A. Einstein, On the quantum theory of radiation, Phys. Z. 18, 121 (1917), http://hermes.ffn.ub.es/luisnavarro/nuevo_ maletin/Einstein\%20(1917)_Quantum\%20theory\%20of $\% 20$ radiation.pdf.

[35] N. Bohr, Física Atómica y Conocimiento Humano (Aguilar, Madrid, 1964), p. 43.

[36] M. Jammer, The Conceptual Development of Quantum Mechanics (Mc Graw Hill, New York, 1966), p. 170-171.

[37] See Ref. [27].

[38] See Ref. [34].

[39] See Ref. [28].

[40] See Ref. [31].

[41] P. A. Tipler and G. Mosca, Physics for Scientists and Engineers, 5th ed. (W. H. Freeman, San Francisco, 2003), Chap. 36.

[42] R. D. Knight, Physics for Scientist and Engineers, 2nd ed. (Benjamin Cummings, San Francisco, 2007), Chap. 38 and Chap. 39.

[43] D. A. Zollman, N. S. Rebello, and K. Hogg, Quantum mechanics for everyone: Hands-on activities integrated with technology, Am. J. Phys. 70, 252 (2002).

[44] V. Sinarcas and J. Solbes, Dificultades en el aprendizaje y la enseñanza de la física cuántica en el bachillerato, Enseñanza de las Ciencias 31, 9 (2013).

[45] L. Ivanjek, P. S. Shaffer, L. C. McDermott, M. Planinic, and D. Veza, Research as a guide for curriculum development: An example from introductory spectroscopy. I. Identifying student difficulties with atomic emission spectra, Am. J. Phys. 83, 85 (2015).
[46] L. Ivanjek, P. S. Shaffer, L. C. McDermott, M. Planinic, and D. Veza, Research as a guide for curriculum development: An example from introductory spectroscopy. II. Addressing students' difficulties with atomic emission spectra, Am. J. Phys. 83, 171 (2015).

[47] B. S. Ambrose, P. R. L. Heron, S. Vokos, and L. C. McDermott, Students understanding of light as an electromagnetic wave: Relating the formalism to physical phenomena, Am. J. Phys. 67, 891 (1999).

[48] H. Fischler and M. Lichfeld, Modern Physics and students' conceptions, Int. J. Sci. Educ. 14, 181 (1992).

[49] M. Budde, H. Niedderer, P. Scott, and J. Leach, 'Electronium': a quantum atomic teaching model, Phys. Educ. 37, 197 (2002).

[50] H. Fischler, Introduction to quantum physics-development and evaluation of a new course, Proceedings of the Annual Meeting of the National Association for Research and Science Teaching (2002), p. 32, http://perg.phys.ksu.edu/ papers/narst/QM_papers.pdf.

[51] REAL DECRETO 1467/2007, de 2 de noviembre, por el que se establece la estructura del bachillerato y se fijan sus enseñanzas mínima. BOE 6 de noviembre de 2007. https://www.boe.es/boe/dias/2007/11/06/pdfs/A45381-45477 .pdf.

[52] J. D. Wilson and J. A. Buffa, College Physics, 3rd ed. (Prentice Hall, New Jersey, 1997), Chap. 27.

[53] See Ref. [44].

[54] See Ref. [45].

[55] See Ref. [43].

[56] See Ref. [45].

[57] See Ref. [45].

[58] See Ref. [49].

[59] See Ref. [50].

[60] See Ref. [47].

[61] See Ref. [48].

[62] See Ref. [30].

[63] G. A. Harrison and D. F. Treagust, A typology of school science models, Int. J. Sci. Educ. 22, 1011 (2000).

[64] See Ref. [43].

[65] See Ref. [45].

[66] F. Halbwachs, Reflexions sur la causalité physique (reflections on causality in physics), in Les Théories de la Causalité, edited by M. Bunge, F. Halbwachs, T. S. Kuhn, J. Piaget, and L. Rosenfeld (Presses Universitaires de France, Paris, 1971), pp. 19-38 and pp. 39-111. 